\title{
A GCD AND LCM-LIKE INEQUALITY FOR MULTIPLICATIVE LATTICES
}

\author{
DANIEL D. ANDERSON, TAKASHI AOKI, SHUZO IZUMI, YASUO OHNO \\ AND MANABU OZAKI
}

\begin{abstract}
Let $A_{1}, \ldots, A_{n}(n \geq 2)$ be elements of an commutative multiplicative lattice. Let $G(k)$ (resp., $L(k)$ ) denote the product of all the joins (resp., meets) of $k$ of the elements.
\end{abstract} Then we show that

$$
L(n) G(2) G(4) \cdots G(2\lfloor n / 2\rfloor) \leq G(1) G(3) \cdots G(2\lceil n / 2\rceil-1) .
$$

In particular this holds for the lattice of ideals of a commutative ring. We also consider the relationship between

$$
G(n) L(2) L(4) \cdots L(2\lfloor n / 2\rfloor) \text { and } L(1) L(3) \cdots L(2\lceil n / 2\rceil-1)
$$

and show that any inequality relationships are possible.

\section{Introduction}

Let $R$ be a commutative ring (not necessarily with identity). Then for two ideals $A_{1}$ and $A_{2}$ of $R$ we have

$$
\left(A_{1} \cap A_{2}\right)\left(A_{1}+A_{2}\right) \subseteq A_{1} A_{2} .
$$

For three ideals $A_{1}, A_{2}, A_{3}$ of $R$ it is easily verified that we have

$$
\left(A_{1} \cap A_{2} \cap A_{3}\right)\left(A_{1}+A_{2}\right)\left(A_{1}+A_{3}\right)\left(A_{2}+A_{3}\right) \subseteq A_{1} A_{2} A_{3}\left(A_{1}+A_{2}+A_{3}\right) .
$$

The purpose of this paper is to give a general containment relation ( $\left.\dagger_{n}\right)$ for $n$ ideals $A_{1}, \ldots, A_{n}$ of $R, n \geq 2$, generalizing the previous two relations $\left(\dagger_{2}\right)$ and $\left(\dagger_{3}\right)$.

The corresponding ideal formulation is as follows. Let $R$ be a commutative ring and let $A_{1}, \ldots, A_{n}(n \geqslant 2)$ be ideals of $R$. For $1 \leqslant k \leqslant n$ put

$$
G(k):=G\left(k ; A_{1}, \ldots, A_{n}\right)=\prod_{1 \leqslant i_{1}<\cdots<i_{k} \leqslant n}\left(A_{i_{1}}+\cdots+A_{i_{k}}\right),
$$

Received January 6, 2015, accepted January 25, 2016.

2010 Mathematics Subject Classification. Primary: 13A15; Secondary: 06F10.

Key words and phrases. Multiplicative lattice, ideals lattice, GCD, LCM.

Corresponding author: Daniel D. Anderson. 


$$
L(k):=L\left(k ; A_{1}, \ldots, A_{n}\right)=\prod_{1 \leqslant i_{1}<\cdots<i_{k} \leqslant n}\left(A_{i_{1}} \cap \cdots \cap A_{i_{k}}\right)
$$

$\left(\right.$ so $\left.G(1)=L(1)=A_{1} \cdots A_{n}, G(n)=A_{1}+\cdots+A_{n}, L(n)=A_{1} \cap \cdots \cap A_{n}\right)$.

Definition 1.1. The ring $R$ satisfies $(*)_{n}$ for ideals $A_{1}, \ldots, A_{n}$ of $R(n \geqslant 2)$ if

$$
G(n) \prod_{2 \leqslant 2 k \leqslant n} L(2 k)=\prod_{1 \leqslant 2 k+1 \leqslant n} L(2 k+1),
$$

satisfies $(* *)_{n}$ for ideals $A_{1}, \ldots, A_{n}$ of $R(n \geqslant 2)$ if

$$
L(n) \prod_{2 \leqslant 2 k \leqslant n} G(2 k)=\prod_{1 \leqslant 2 k+1 \leqslant n} G(2 k+1),
$$

and satisfies $\left(\dagger_{n}\right)$ for ideals $A_{1}, \ldots, A_{n}$ of $R(n \geq 2)$ if

$$
L(n) \prod_{2 \leq 2 k \leq n} G(2 k) \subseteq \prod_{1 \leq 2 k+1 \leq n} G(2 k+1) .
$$

Using the ceiling function and the floor function, we may express these as follows:

$$
\begin{aligned}
& G(n) L(2) L(4) \cdots L(2\lfloor n / 2\rfloor)=L(1) L(3) \cdots L(2\lceil n / 2\rceil-1), \\
& L(n) G(2) G(4) \cdots G(2\lfloor n / 2\rfloor)=G(1) G(3) \cdots G(2\lceil n / 2\rceil-1), \\
& L(n) G(2) G(4) \cdots G(2\lfloor n / 2\rfloor) \subseteq G(1) G(3) \cdots G(2\lceil n / 2\rceil-1) .
\end{aligned}
$$

Note that $(*)_{2}$ reduces to $\left(A_{1}+A_{2}\right)\left(A_{1} \cap A_{2}\right)=A_{1} A_{2}$ and $(* *)_{2}$ reduces to $\left(A_{1} \cap A_{2}\right)\left(A_{1}+\right.$ $\left.A_{2}\right)=A_{1} A_{2}$ while as previously mentioned $\left(\dagger_{2}\right)$ is $\left(A_{1} \cap A_{2}\right)\left(A_{1}+A_{2}\right) \subseteq A_{1} A_{2}$. We are taking $n \geqslant 2$ as the properties $(*)_{1}$ and $(* *)_{1}$ are simply $A_{1}=A_{1}$ which is always true as is $\left(\dagger_{1}\right) A_{1} \subseteq A_{1}$.

A commutative ring $R$ is called a chained ring (resp., arithmetical ring) if the lattice of ideals of $R$ is a chain (resp., distributive). So an integral domain is a chained ring if and only if it is a valuation domain. It is well known that $R$ is an arithmetical ring if and only if $R_{M}$ is a chained ring for each maximal ideal $M$ of $R$. An integral domain is a Prüfer domain if every nonzero finitely generated ideal is invertible. $R$ is a Prüfer domain if and only if $R_{M}$ is a valuation domain for each maximal ideal $M$ of $R$ if and only if $R_{M}$ is a chained ring for each maximal ideal $M$ of $R$. Thus a Prüfer domain is an arithmetical ring that is an integral domain. Finally, $R$ is a Prüfer ring if every finitely generated regular ideals is invertible. Here, an element is regular if it is not a zero-divisor and an ideal is regular if it contains a regular element.

We showed [1, Theorem 2.4] if $R$ is an arithmetical ring, then $(*)_{n}$ and $(* *)_{n}$ hold for all ideals $A_{1}, \ldots, A_{n}$ of $R$ and that, $R$ is a Prüfer ring if and only if $(* *)_{n}$ hold for some $n \geq 2$ 
(equivalently, for all $n \geq 2$ ) for all ideals $A_{1}, \ldots, A_{n}$ of $R$ when at least $n-1$ of them are regular [1, Theorem 2.6]. We also proved that $(\mathrm{GCD})_{n}$ and (LCM) $)_{n}$ hold for any GCD domain $[1$, Theorem 2.8]:

$$
\begin{gathered}
\operatorname{gcd}\left(a_{1}, \ldots, a_{n}\right) \prod_{2 \leqslant 2 k \leqslant n 1 \leqslant i_{1}<\cdots<i_{2 k} \leqslant n} \operatorname{lcm}\left(a_{i_{1}}, \ldots, a_{i_{2 k}}\right) \\
\quad=a_{1} \cdots a_{n} \prod_{2 \leqslant 2 k+1 \leqslant n} \prod_{1 \leqslant i_{1}<\cdots<i_{2 k+1} \leqslant n} \operatorname{lcm}\left(a_{i_{1}}, \ldots, a_{i_{2 k+1}}\right) \\
\operatorname{lcm}\left(a_{1}, \ldots, a_{n}\right) \prod_{2 \leqslant 2} \prod_{k \leqslant n} \prod_{1 \leqslant i_{1}<\cdots<i_{2 k} \leqslant n} \operatorname{gcd}\left(a_{i_{1}}, \ldots, a_{i_{2 k}}\right) \\
\quad=a_{1} \cdots a_{n} \prod_{2 \leqslant 2 k+1 \leqslant n} \prod_{1 \leqslant i_{1}<\cdots<i_{2 k+1} \leqslant n} \operatorname{gcd}\left(a_{i_{1}}, \ldots, a_{i_{2 k+1}}\right) .
\end{gathered}
$$

Note that for a PID $R, \mathrm{GCD}_{n}$ (resp., $\mathrm{LCM}_{n}$ ) may be obtained from $(*)_{n}$ (resp., $(* *)_{n}$ ) by taking $A_{1}=\left(a_{1}\right), \ldots, A_{n}=\left(a_{n}\right)$.

Thus neither $(*)_{n}$ nor $(* *)_{n}$ always holds. In Section 2 , however, we show that the onesided inclusion

$$
L(n) \prod_{2 \leqslant 2 k \leqslant n} G(2 k) \subseteq \prod_{1 \leqslant 2 k+1 \leqslant n} G(2 k+1)
$$

holds for general commutative rings (which may not have an identity). Indeed, this holds not only for ideal lattices of commutative rings, but in the quite general setting of a (commutative) multiplicative lattice. In Section 3 we give some examples to illustrate results from Section 2.

\section{Inclusion Formula for Multiplicative Lattices}

We have noted in the Introduction that the identity $(*)_{n}$ or $(* *)_{n}$ holds for all ideals of special rings. However one inclusion formula holds for a general commutative ring as follows. Using the expression in the former section, it is expressed as

$$
\begin{array}{r}
L\left(n ; A_{1}, A_{2}, \ldots, A_{n}\right) \prod_{2 \leqslant 2 k \leqslant n} G\left(2 k ; A_{1}, A_{2}, \ldots, A_{n}\right) \\
\subseteq \prod_{1 \leqslant 2 k+1 \leqslant n} G\left(2 k+1 ; A_{1}, A_{2}, \ldots, A_{n}\right)
\end{array}
$$

or equivalently,

$$
L(n) G(2) G(4) \cdots G(2\lfloor n / 2\rfloor) \subseteq G(1) G(3) \cdots G(2\lceil n / 2\rceil-1) .
$$

This is the only inclusion formula concerning both sides of $(*)_{n}$ and $(* *)_{n}$ which holds for all ideals of a general commutative ring. For, it is shown by Example 3.1, that the opposite inclusion does not always hold and by Example 3.2 that neither of the inclusions between 
$G(n) L(2) L(4) \cdots L(2\lfloor n / 2\rfloor)$ and $L(1) L(3) \cdots L(2\lceil n / 2\rceil-1)$ always holds. We prove the above inclusion in a more generalized form: inequality in a (commutative) multiplicative lattice.

By a multiplicative lattice we mean a lattice with a commutative, associative product that distributes over finite joins. Observe that $A \leq B$ implies $A C \leq B C$ for elements $A, B$, and $C$ of a multiplicative lattice. The ideals of a commutative ring (or even a semiring) or commutative multiplicative semigroup with 0 forms a complete multiplicative lattice with $A \vee B=A+B$ for a ring or semiring ( $A \vee B=A \cup B$ for a semigroup), $A \wedge B=A \cap B$, and $A B$ as the usual ideal product.

Given elements $A_{1}, \ldots, A_{n}$ of a multiplicative lattice $\mathscr{I}$, we can define $G(k), L(k) \in \mathscr{I}$ :

$$
\begin{aligned}
G(k) & :=\prod_{1 \leqslant i_{1}<\cdots<i_{k} \leqslant n}\left(A_{i_{1}} \vee \cdots \vee A_{i_{k}}\right), \\
L(k) & :=\prod_{1 \leqslant i_{1}<\cdots<i_{k} \leqslant n}\left(A_{i_{1}} \wedge \cdots \wedge A_{i_{n}}\right),
\end{aligned}
$$

as in Section 1, replacing + and $\cap$ respectively by $\vee$ and $\wedge$. The identities $(*)_{n},(* *)_{n}$ and $\left(\dagger_{n}\right)$ are defined in the same way as Definition 1.1. Then we can prove the following generalization of [1, Lemma 2.1]. The proof is similar.

Proposition 2.1. Let $\mathscr{I}$ be a multiplicative lattice and take $A_{1}, \ldots, A_{n} \in \mathscr{I}(n \geqslant 2)$. Suppose that $\left\{A_{1}, \ldots, A_{n}\right\}$ has a maximum (resp., minimum) element. Then $(*)_{n}\left(\right.$ resp., $\left.(* *)_{n}\right)$ holds for $A_{1}, \ldots, A_{n} \in \mathscr{I}$.

In this general setting, we do not know any other meaningful sufficient condition for the identities $(*)_{n}$ and $(* *)_{n}$ to hold. Thus we content ourselves with a one-sided inequality as follows. Note that it implies the one-sided inclusion formula for ideals of a general commutative semiring (and hence ring) which may not have an identity.

Theorem 2.2. Let $\mathscr{I}$ be a multiplicative lattice. For $A_{1}, \ldots, A_{n} \in \mathscr{I}(n \in \mathbb{N})$, we always have the following:

$$
L(n) G(2) G(4) \cdots G(2\lfloor n / 2\rfloor) \leqslant G(1) G(3) \cdots G(2\lceil n / 2\rceil-1) .
$$

Proof. In this theorem, $\left(\dagger_{1}\right)$ should be interpreted as the trivial assertion $A \leqslant A$. The assertion $\left(\dagger_{2}\right)$ follows from

$$
\left(A_{1} \wedge A_{2}\right)\left(A_{1} \vee A_{2}\right) \leqslant A_{2} A_{1} \vee A_{1} A_{2}=A_{1} A_{2}
$$

Assume that, for some $n \geqslant 3$, we have proved $\left(\dagger_{k}\right)(k<n)$. Let us put

$$
G\left(p ; q, r ; A_{1}, \ldots, A_{n}\right):=\prod_{q<i_{1}<\cdots<i_{p-2}<r}\left(A_{q} \vee A_{i_{1}} \vee \cdots \vee A_{i_{p-2}} \vee A_{r}\right)
$$




$$
(1 \leqslant q \leqslant r \leqslant n, 1 \leqslant p \leqslant r-q+1) .
$$

Here, in the case $r=q$,

$$
G\left(1 ; q, q ; A_{1}, \ldots, A_{n}\right)=A_{q},
$$

and in the case $r=q+1$,

$$
\begin{aligned}
& G\left(1 ; q, q+1 ; A_{1}, \ldots, A_{n}\right)=A_{q} A_{q+1}, \\
& G\left(2 ; q, q+1 ; A_{1}, \ldots, A_{n}\right)=A_{q} \vee A_{q+1} .
\end{aligned}
$$

We also have

$$
G\left(p ; A_{1}, \ldots, A_{n}\right)=\prod_{\substack{1 \leqslant q \leqslant n \\ p+q-1 \leqslant r \leqslant n}} G\left(p ; q, r ; A_{1}, \ldots, A_{n}\right)
$$

for $1 \leqslant p \leqslant n$.

If $n$ is even: $n=2 m \geqslant 4$, we have to prove

$$
\begin{aligned}
\text { (Left) }) & :=L\left(2 m ; A_{1}, \ldots, A_{2 m}\right) \prod_{1 \leqslant p \leqslant m} G\left(2 p ; A_{1}, \ldots, A_{2 m}\right) \\
& \leqslant \prod_{1 \leqslant p \leqslant m} G\left(2 p-1 ; A_{1}, A_{2}, \ldots, A_{2 m}\right)=: \text { (Right). }
\end{aligned}
$$

The expression (Left) contains the factor $\left(A_{2 m-1} \vee A_{2 m}\right)$. Let (Left) $2 m-1$ (resp., (Left) $2 m$ ) denote the expression obtained by substitution of this factor $\left(A_{2 m-1} \vee A_{2 m}\right)$ by $A_{2 m-1}$ (resp. by $A_{2 m}$ ) in $($ Left $)$. Since $($ Left $)=(\text { Left })_{2 m-1} \vee(\text { Left })_{2 m}$, we only have to prove

$$
\text { (Left) })_{2 m-1} \leqslant \text { (Right), (Left) } 2 m \leqslant \text { (Right). }
$$

By symmetry, we only have to prove the latter. Since

$$
\begin{aligned}
(\text { Left })= & L\left(2 m ; A_{1}, \ldots, A_{2 m}\right) \prod_{1 \leqslant p \leqslant m-1}\left(G\left(2 p ; A_{1}, \ldots, A_{2 m-1}\right)\right. \\
& \left.\prod_{1 \leqslant q \leqslant 2 m-2 p+1} G\left(2 p ; q, 2 m ; A_{1}, \ldots, A_{2 m}\right)\right) \\
= & L\left(2 m ; A_{1}, \ldots, A_{2 m}\right)\left(\prod_{1 \leqslant p \leqslant m-1} G\left(2 p ; A_{1}, \ldots, A_{2 m-1}\right)\right) \\
& \cdot\left(\prod_{1 \leqslant q \leqslant 2 m-1}\left(A_{q} \vee A_{2 m}\right)\right)\left(\prod_{\substack{1 \leqslant q \leqslant 2 m-2 p+1 \\
2}} G\left(2 p ; q, 2 m ; A_{1}, \ldots, A_{2 m}\right)\right) \\
= & L\left(2 m ; A_{1}, \ldots, A_{2 m}\right)\left(\prod_{1 \leqslant p \leqslant m-1} G\left(2 p ; A_{1}, \ldots, A_{2 m-1}\right)\right) \cdot\left(\prod_{1 \leqslant q \leqslant 2 m-1}\left(A_{q} \vee A_{2 m}\right)\right) \\
& \cdot\left(\prod _ { \substack { 2 \leqslant p \leqslant m \\
1 \leqslant q \leqslant 2 m - 2 p + 1 } } G \left(2 p-2 ; A_{q} \vee A_{q+1} \vee A_{2 m},\right.\right.
\end{aligned}
$$




$$
\left.\left.A_{q} \vee A_{q+2} \vee A_{2 m}, \ldots, A_{q} \vee A_{2 m-1} \vee A_{2 m}\right)\right)
$$

if we put

$$
B_{q, v}:=A_{q} \vee A_{v} \vee A_{2 m} \quad(q<v<2 m),
$$

we have

$$
\begin{aligned}
(\text { Left })_{2 m}= & L\left(2 m ; A_{1}, \ldots, A_{2 m}\right)\left(\prod_{1 \leqslant p \leqslant m-1} G\left(2 p ; A_{1}, \ldots, A_{2 m-1}\right)\right) \cdot\left(\prod_{1 \leqslant q \leqslant 2 m-2}\left(A_{q} \vee A_{2 m}\right)\right) A_{2 m} \\
& \left(\prod_{\substack{2 \leqslant p \leqslant m \\
1 \leqslant q \leqslant 2 m-2 p+1}} G\left(2 p-2 ; B_{q, q+1}, B_{q, q+2}, \ldots, B_{q, 2 m-1}\right)\right) .
\end{aligned}
$$

Note that

$$
A_{q} \vee A_{2 m} \leqslant L\left(2 m-q-1 ; B_{q, q+1}, B_{q, q+2}, \ldots, B_{q, 2 m-1}\right)
$$

Thus we have

$$
\begin{aligned}
(\text { Left })_{2 m}= & L\left(2 m ; A_{1}, \ldots, A_{2 m}\right)\left(\prod_{1 \leqslant p \leqslant m-1} G\left(2 p ; A_{1}, \ldots, A_{2 m-1}\right)\right) \cdot\left(A_{2 m-2} \vee A_{2 m}\right) A_{2 m} \\
& \cdot \prod_{1 \leqslant q \leqslant 2 m-3}\left(\left(A_{q} \vee A_{2 m}\right) \prod_{2 \leqslant p \leqslant m-(q-1) / 2} G\left(2 p-2 ; B_{q, q+1}, B_{q, q+2}, \ldots, B_{q, 2 m-1}\right)\right) \\
\leqslant & L\left(2 m-1 ; A_{1}, \ldots, A_{2 m-1}\right)\left(\prod_{1 \leqslant p \leqslant m-1} G\left(2 p ; A_{1}, \ldots, A_{2 m-1}\right)\right) \cdot\left(A_{2 m-2} \vee A_{2 m}\right) A_{2 m} \\
& \cdot \prod_{1 \leqslant q \leqslant 2 m-3}\left(L\left(2 m-q-1 ; B_{q, q+1}, \ldots, B_{q, 2 m-1}\right)\right. \\
& \left.\quad \prod_{2 \leqslant p \leqslant m+(1-q) / 2} G\left(2 p-2 ; B_{q, q+1}, B_{q, q+2}, \ldots, B_{q, 2 m-1}\right)\right) .
\end{aligned}
$$

By the inductive assumption and by

$$
A_{2 m-2} \vee A_{2 m} \leqslant A_{2 m-2} \vee A_{2 m-1} \vee A_{2 m}
$$

the expression (Left) $2 m$ is majorized by

$$
\begin{aligned}
& \left(\prod_{1 \leqslant p \leqslant m} G\left(2 p-1 ; A_{1}, \ldots, A_{2 m-1}\right)\right)\left(A_{2 m-2} \vee A_{2 m-1} \vee A_{2 m}\right) A_{2 m} \\
& \quad \cdot \prod_{1 \leqslant q \leqslant 2 m-3} \prod_{1 \leqslant p \leqslant m-q / 2} G\left(2 p-1 ; B_{q, q+1}, B_{q, q+2}, \ldots, B_{q, 2 m-1}\right) \\
& \quad \leqslant\left(\prod_{1 \leqslant p \leqslant m} G\left(2 p-1 ; A_{1}, \ldots, A_{2 m}\right)\right)=(\text { Right }) .
\end{aligned}
$$

Here note that $A_{2 m-2} \vee A_{2 m-1} \vee A_{2 m}$ is the only join of three $A_{i}$ 's which contains $A_{2 m}$ and is not equal to some $B_{q, r}$. 
In the case $n=2 m+1 \geqslant 3$, putting $C_{q, r}:=A_{q} \vee A_{r} \vee A_{2 m+1}$ and replacing the factor $A_{2 m} \vee$ $A_{2 m+1}$ of

$$
\text { (Left) }:=L\left(2 m+1 ; A_{1}, \ldots, A_{2 m+1}\right) \prod_{1 \leqslant p \leqslant m} G\left(2 p ; A_{1}, \ldots, A_{2 m+1}\right)
$$

by $A_{2 m+1}$, we similarly have the following.

$$
\begin{aligned}
(\text { Left })_{2 m+1}= & L\left(2 m ; A_{1}, \ldots, A_{2 m+1}\right)\left(\prod_{1 \leqslant p \leqslant m} G\left(2 p ; A_{1}, \ldots, A_{2 m}\right)\right) \\
& \cdot\left(A_{2 m-1} \vee A_{2 m+1}\right) A_{2 m+1} \\
& \cdot \prod_{1 \leqslant q \leqslant 2 m-2}\left(L\left(2 m-q ; C_{q, q+1}, \ldots, C_{q, 2 m}\right)\right. \\
& \left.\prod_{2 \leqslant p \leqslant m+1-q / 2} G\left(2 p-2 ; C_{q, q+1}, C_{q, q+2}, \ldots, C_{q, 2 m}\right)\right) \\
\leqslant & \left(\prod_{1 \leqslant p \leqslant m} G\left(2 p-1 ; A_{1}, \ldots, A_{2 m}\right)\right)\left(A_{2 m-1} \vee A_{2 m} \vee A_{2 m+1}\right) A_{2 m+1} \\
& \cdot \prod_{1 \leqslant q \leqslant 2 m-21 \leqslant p \leqslant m} G\left(2 p-1 ; C_{q, q+1}, C_{q, q+2}, \ldots, C_{q, 2 m}\right) \\
= & \left(\prod_{1 \leqslant p \leqslant m} G\left(2 p-1 ; A_{1}, \ldots, A_{2 m+1}\right)\right)=(\text { Right }) .
\end{aligned}
$$

This completes the mathematical induction.

\section{Examples}

In this section we give some examples which illustrate the results of Section 2.

Example 3.1 (A ring with $L(n) \prod_{2 \leqslant 2 k \leqslant n} G(2 k) \subsetneq \prod_{1 \leqslant 2 k+1 \leqslant n} G(2 k+1)$ for all $n \geqslant 2$ ). Put $R=$ $k\left[X_{1}, X_{2}, \ldots\right]$ for a field $k$ and take $A_{i}=\left(X_{i}\right)$. Here $L(n)=\left(X_{1}\right) \cap \cdots \cap\left(X_{n}\right)=\left(X_{1} \cdots X_{n}\right), G(1)=$ $\left(X_{1} \cdots X_{n}\right)$ and $G(n)=\left(X_{1}, \ldots, X_{n}\right)$. We can quote Theorem 2.2 to get

$$
L(n) \prod_{2 \leqslant 2 k \leqslant n} G(2 k) \subseteq \prod_{1 \leqslant 2 k+1 \leqslant n} G(2 k+1) .
$$

However, we sketch a proof. This example shows that the inequality given in Theorem 2.2 may be strict, or equivalently, that the reverse inequality need not hold.

Note that each of the ideals $L(n)$ and $G(l)(1 \leqslant l \leqslant n)$ is generated by monomials in $X_{1}, \ldots, X_{n}$. Of course $L(n)$ and $G(1)$ are generated by $X_{1} \cdots X_{n}$ and $G(n)$ is generated by $X_{1}, \ldots, X_{n}$. Suppose $1<k<n, G(k)=\prod_{1 \leqslant i_{1}<\cdots<i_{k} \leqslant n}\left(X_{i_{1}}, \ldots, X_{i_{n}}\right)$, a product of $\left(\begin{array}{l}n \\ k\end{array}\right)$ ideals and hence is generated by monomials in $X_{1}, \ldots, X_{n}$ of degree $\left(\begin{array}{l}n \\ k\end{array}\right)$. In fact $G(k)$ is generated by

$$
\left\{X_{1}^{\alpha_{1}} \cdots X_{n}^{\alpha_{n}}: 0 \leqslant \alpha_{i} \leqslant\left(\begin{array}{l}
n-1 \\
k-1
\end{array}\right), \alpha_{1}+\cdots+\alpha_{n}=\left(\begin{array}{l}
n \\
k
\end{array}\right)\right\} .
$$


Suppose $n$ is even. Then $L(n) \prod G(2 k)$ is generated by the monomials

$$
X_{1} \cdots X_{n} \prod_{1 \leqslant k \leqslant n / 2-1} X_{1}^{\alpha_{1,2 k}} \cdots X_{n}^{\alpha_{n, 2 k}} X_{1}^{\beta_{1}} \cdots X_{n}^{\beta_{n}}
$$

where

$$
\begin{gathered}
0 \leqslant \alpha_{i, 2 k} \leqslant\left(\begin{array}{c}
n-1 \\
2 k-1
\end{array}\right), \quad \alpha_{1,2 k}+\cdots+\alpha_{n, 2 k}=\left(\begin{array}{c}
n \\
2 k
\end{array}\right), \\
0 \leqslant \beta_{i} \leqslant 1, \quad \beta_{1}+\cdots+\beta_{n}=1 .
\end{gathered}
$$

This may be rewritten as $\prod_{1 \leqslant i \leqslant n} X_{i}^{1+\alpha_{i, 2}+\cdots+\alpha_{i, n-2}+\beta_{i}}$ and has degree $n+\sum_{1 \leqslant k \leqslant n / 2}\left(\begin{array}{c}n \\ 2 k\end{array}\right)$. Similarly one can write down the generators for $G(2 k+1)$ which are monomials of degree $\sum_{0 \leqslant k \leqslant n / 2-1}\left(\begin{array}{c}n \\ 2 k+1\end{array}\right)$. Now

$$
n+\sum_{1 \leqslant k \leqslant n / 2}\left(\begin{array}{c}
n \\
2 k
\end{array}\right)=\sum_{0 \leqslant k \leqslant n / 2-1}\left(\begin{array}{c}
n \\
2 k+1
\end{array}\right),
$$

so the monomial generators for $L(n) \prod G(2 k)$ have degree $n-1$ greater than the ones for $G(2 k+$ 1 ), which rules out equality of $L(n) \prod G(2 k)$ and $\prod G(2 k+1)$. A rather messy argument shows that each of the monomial generators for $L(n) \prod G(2 k)$ is a multiple of a monomial generator for $\prod G(2 k+1)$. Thus we have $L(n) \prod G(2 k) \subsetneq \prod G(2 k+1)$. The case for $n$ odd is similar.

Example 3.2 (Any relationship between $G(3) L(2)$ and $L(1) L(3)$ is possible). Take $R=k[X, Y, Z]$, $k$ a field.

1. $G(3) L(2)=L(1) L(3)$ : Take $A_{1}=(X), A_{2}=(Y), A_{3}=(X, Y)$. Since $A_{1}, A_{2} \subseteq A_{3}, G(3) L(2)=$ $L(1) L(3)$ by Proposition 2.1 or [1, Lemma 2.1].

2. $G(3) L(2) \subsetneq L(1) L(3)$ : Take $A_{1}=(X), A_{2}=(Y), A_{3}=(Z)$. By [1, Example 3.3], $G(3) L(2) \subsetneq$ $L(1) L(3)$.

3. $G(3) L(2) \supsetneq L(1) L(3):$ Take

$$
A_{1}=(X, Y), \quad A_{2}=(Y, Z), \quad A_{3}=(Z, X)
$$

So $G(3)=(X, Y, Z)$ and

$$
\begin{aligned}
L(2)=((X, Y) & \cap(X, Z))((X, Y) \cap(X, Z))((X, Z) \cap(Y, Z)) \\
& =(X, Y Z)(Y, X Z)(Z, X Y) .
\end{aligned}
$$

So

$$
\begin{gathered}
G(3) L(2)=(X, Y, Z)(X, Y Z)(Y, X Z)(Z, X Y) \\
=\left(X^{3} Y^{2}, X^{2} Y^{3}, Y^{3} Z^{2}, Y^{2} Z^{3}, X^{2} Z^{3}, X^{3} Z^{2}, X^{2} Y Z, X Y^{2} Z, X Y Z^{2}\right) .
\end{gathered}
$$


On the other hand,

$$
\begin{gathered}
L(1) L(3)=(X, Y)(Y, Z)(Z, X)((X, Y) \cap(Y, Z) \cap(Z, X)) \\
=(X, Y)(Y, Z)(Z, X)(X Y, Y Z, Z X) \\
=\left(X^{3} Y^{2}, X^{2} Y^{3}, Y^{3} Z^{2}, Y^{2} Z^{3}, X^{2} Z^{3}, X^{3} Z^{2}\right. \\
\left.X^{2} Y^{2} Z, X^{2} Y Z^{2}, X Y^{2} Z^{2}, X^{3} Y Z, X Y^{3} Z, X Y Z^{3}\right),
\end{gathered}
$$

which is easily checked to be a proper subset of $G(3) L(2)$.

4. $G(3) L(2)$ and $L(1) L(3)$ are incomparable: Take ideals

$$
A_{1}=\left(X^{2}, Y\right), \quad A_{2}=\left(Y^{3}, Z\right), \quad A_{3}=(X Y, Z) .
$$

First we show $X Y^{5} Z \in G(3) L(2)$ but $X Y^{5} Z \notin L(1) L(3)$. The first inclusion follows from

$$
\begin{gathered}
X Y^{5} Z=Y \cdot Y^{3} \cdot Z \cdot X Y \\
\in\left(A_{1}+A_{2}+A_{3}\right)\left(A_{1} \cap A_{2}\right)\left(A_{2} \cap A_{3}\right)\left(A_{1} \cap A_{3}\right) .
\end{gathered}
$$

On the other hand,

$$
\begin{gathered}
A_{1} \cap A_{2} \cap A_{3}=\left(A_{1} \cap A_{2}\right) \cap A_{3} \\
=\left(X^{2} Z, Y^{3}, Y Z\right) \cap(X Y, Z)=\left(X^{2} Z, X Y^{3}, Y Z\right) .
\end{gathered}
$$

If

$$
X Y^{5} Z \in L(1) L(3)=A_{1} A_{2} A_{3}\left(A_{1} \cap A_{2} \cap A_{3}\right),
$$

it follows that $X Y^{5} Z \in A_{1} A_{2} A_{3}\left(X Y^{3}, Y Z\right)$. So $Y^{2} Z \in A_{1} A_{2} A_{3}$ or $X Y^{4} \in A_{1} A_{2} A_{3}$. This implies $Y^{2} Z \in(Y)(Z)(Z)$ or $X Y^{4} \in\left(X^{2}, Y\right)\left(Y^{3}\right)(X Y)$, each of which obviously yields a contradiction. Thus we have proved that $X Y^{5} Z \notin L(1) L(3)$.

Next we prove that $Y^{2} Z^{3} \in L(1) L(3)$ but $Y^{2} Z^{3} \notin G(3) L(2)$. The first inclusion follows from

$$
Y^{2} Z^{3}=Y \cdot Z \cdot Z \cdot Y Z \in A_{1} A_{2} A_{3}\left(A_{1} \cap A_{2} \cap A_{3}\right) .
$$

Since each nonzero element of

$$
\begin{gathered}
L(2)=\left(X^{2} Z, Y^{3}, Y Z\right)\left(X Y^{3}, Z\right)\left(X^{2} Z, X Y, Y Z\right) \\
\text { (resp., } \left.G(3)=\left(X^{2}, Y, Z\right)\right)
\end{gathered}
$$

has degree at least 5 (resp., 1), any nonzero element of $G(3) L(2)$ has degree at least 6 . Hence $Y^{2} Z^{3}$ can not be contained in $G(3) L(2)$.

\section{Acknowledgement}

We thank Yayoi Nakamura for confirmation of some facts using CAS. 


\section{References}

[1] D. D. Anderson, S. Izumi, Y. Ohno and M. Ozaki, GCD and LCM-like identities for ideals in commutative rings, J. Algebra Appl., 15 (2016), 1650010 (12 pages).

Department of Mathematics, The University of Iowa, Iowa City, IA 52242, USA.

E-mail: dan-anderson@uiowa.edu

Department of Mathematics, Kindai University, Higashi-Osaka, 577-8502, Japan.

E-mail: aoki@math.kindai.ac.jp

Department of Mathematics, Kindai University, Higashi-Osaka, 577-8502, Japan.

E-mail: sizmsizm@gmail.com

Mathematical Institute, Tohoku University, Sendai, 980-8578, Japan.

E-mail: ohno@math.tohoku.ac.jp

Department of Mathematics, Waseda University, Shinjuku-ku Tokyo.

E-mail: ozaki@waseda.jp 